\title{
Human and Rabbit Newborn Lung Macrophages Have Reduced Anti-Candida Activity
}

\author{
JOHN B. D'AMBOLA, MICHAEL P. SHERMAN, DONALD P. TASHKIN, AND HENRY GONG, JR. \\ Division of Neonatology, Department of Pediatrics [J.B.D., M.P.S.] and Pulmonary Division, Department of \\ Medicine [D.P.T., H.G.], University of California at Los Angeles, Los Angeles, California 90024
}

\begin{abstract}
Bronchoalveolar macrophages (BAM) protect the adult lung from low level microbial contamination. The antimicrobial activity of human newborn BAM is unknown. BAM were isolated from effluents of suctioned, intubated newborns and from bronchoalveolar lavage of healthy, nonsmoking adult volunteers. An in vitro cytologic slide assay was developed and used to ascertain: 1) inhibition of intracellular filamentation of Candida albicans (active yeast growth) and 2) killing + digestion of ingested C. albicans. The ability to restrict intracellular yeast filamentation was markedly different for human newborn versus adult BAM. Adult BAM were five times more effective in restricting intracellular filamentation of Candida compared to newborn cells $(\boldsymbol{p}<0.01)$. Nonfilamented ingested yeast were also handled differently by newborn and adult macrophages. Nonfilamented yeast were killed and digested by adult BAM at a rate that was 2.5 times above that noted in neonatal lung macrophages $(p<0.005)$. However, no differences were found in the total number of killed + digested Candida within human newborn and adult BAM [adult $=32.4 \pm 10.5 \%(n=5)$, newborn $<1200 \mathrm{~g}=$ $39.6 \pm 16.8 \%(n=8)$, and newborn $>1200 \mathrm{~g}=30.2 \pm$ $11.1 \%(n=16)$, mean \pm S.D.]. Neonatal BAM were able to destroy $C$. albicans at a level equivalent to adult cells because these newborn phagocytes allowed intracellular Candida to enter a state of active growth, thereby rendering the yeast more susceptible to killing and digestion. The anti-Candida activity noted in lung macrophages recovered from normal 1-day-old and adult rabbits was similar to that seen in human BAM. Kinetic studies of intra- and extracellular yeast filamentation with time revealed that extracellular yeast were unrestricted in their ability to grow (filamentation $=98 \pm 0.5 \%$ by $2 \mathrm{~h}$ ). Bonchoalveolar macrophages recovered from rabbits exposed to $95+\% \mathrm{O}_{2}$ for $96 \mathrm{~h}$ after birth had a 2 -fold higher rate of intracellular Candida filamentation when compared to BAM lavaged from newborn rabbits raised in room air $[66.3 \pm 5.9 \%$ versus $33 \pm 13.5 \%$ (mean $\pm \mathrm{SD}, p<0.025$ )]. Oxygen exposure did not alter macrophage phagocytosis. We conclude that oxygen therapy may be an important factor underlying the fungistatic limitations of BAM from newborns undergoing intensive care. (Pediatr Res 24: 285-290, 1988)
\end{abstract}

Abbreviations

BAM, bronchoalveolar macrophage BSH, balanced salt-HEPES buffer

Received September 1, 1987; accepted April 20, 1988

Correspondence and reprint requests John B. D'Ambola, M.D., Department of Pediatrics, Olive View Medical Center, Room 3A108, 14445 Olive View Drive Sylmar, CA 91342.

Supported by Grants HL 40675, DA 03018, and HL 35640 from the National Institutes of Health and U.S. Public Health Service CRC Grant RR 00865.
The lung is the most common organ-specific site of nosocomial infections in pediatric patients (1). Neonates who develop bronchopulmonary dysplasia during their recovery from the respiratory distress syndrome are particularly susceptible to infectious pneumonia $(2,3)$. Animal studies indicate that the adult lung has a fully established bronchoalveolar macrophage system that effectively clears the air spaces from microbial contamination compared to the neonate $(4,5)$. Because no studies have examined the antimicrobial function of human newborn BAM, we report a technique that was able to use limited numbers of cells and ascertain the anti-Candida activity of neonatal lung macrophages compared to their adult counterparts.

\section{METHODS}

Patients. Fourteen preterm and 10 term infants requiring intubation and mechanical ventilation as part of their care at the UCLA Medical Center were the subjects of the newborn part of this study. Birth weight, gestational age, and relevant clinical diagnoses were based on data in the hospital record. Neonatal patients ranged from 3 days to 4 months of age at the time of study. No neonatal subjects had a diagnosis of acute bacterial infection of the lung when there were examined. Five healthy, nonsmoking volunteers (three male and two female, $27 \pm 7 \mathrm{yr}$ ) free of respiratory disease and drug or medication intake for the proceeding month, were the subject for the adult phase of this investigation.

Collection and preparation of bronchoalveolar macrophages. Human newborn pulmonary macrophages were obtained from intubated infants by collecting material normally discarded as part of routine suctioning. Before suctioning, $0.25 \mathrm{ml}$ of sterile, nonbacteriostatic saline was instilled into the endotracheal tube to facilitate removal of the tracheal debris. Suctioned material was collected into sterile specimen traps containing $10 \mathrm{ml}$ of RPMI and $2 \mathrm{ml}$ of $20 \% \mathrm{~N}$-acetyl-L-cysteine solution (Mucomyst). The RPMI contained streptomycin $(10,000 \mu \mathrm{g} / \mathrm{ml})$ and penicillin $(10,000 \mathrm{IU} / \mathrm{ml})$ to inhibit bacterial growth. Material from three to five suctionings, over a 4- to 6-h period, was collected into a single specimen trap that was kept in an ice water bath. The collected material was filtered through two layers of sterile gauze to remove any large clumps of mucus, and spun at low speed $(150 \times g)$ to isolate a cell pellet. The supernatant was discarded and the cell pellet resuspended in BSH (6). The cells were counted in a hemocytometer using $0.4 \%$ trypan blue in Dulbecco's phosphate-buffered saline as the diluent. Only specimens containing BAM having a viability $\geq 90 \%$ (trypan blue exclusion) were studied. The final concentration of cells was adjusted to $5 \times 10^{6}$ viable $\mathrm{BAM} / \mathrm{ml}$.

After informed consent of the adult human subjects, their BAM were recovered by fiberoptic bronchoscopy and bronchoalveolar lavage as previously described (7). The lavage effluent was centrifuged and the cell pellet resuspended in RPMI and kept in an ice water bath for 4-6 h. Thereafter, the cells were recentrifuged and suspended in BSH, counted (trypan blue exclusion $\geq 90 \%$ ), and adjusted to a final concentration of $5 \times 10^{6}$ viable 
BAM $/ \mathrm{ml}$. Therefore, except for the presence of Mucomyst the adult and newborn macrophages were processed in an identical manner. Protocols for these investigations were approved by our institutional review committee for studies involving human subjects.

Preparation of Candida. Candida albicans strain 820 was grown to log phase $\left(16 \mathrm{~h}, 37^{\circ} \mathrm{C}\right)$ in Sabouraud $2 \%$ dextrose broth (Difco Laboratories, Detroit, MI). After washing by centrifugation in sterile normal saline, the resuspended yeast were held in an ice bath and sonicated with a microprobe (Biosonik IV, Bronwill, VWR Scientific, San Francisco, CA) at $70 \%$ power level for three 7-s intervals with 30 -s rest periods between disruptions. The Candida were counted using sterile saline containing $0.01 \%$ methylene blue. Methylene blue exclusion was used as a preliminary measure of yeast viability $(\geq 99 \%)(8)$.

In vitro anti-Candida assay. A total of $1 \times 10^{5}(20 \mu \mathrm{l})$ cells from a newborn or adult suspension were mixed with $6.5 \mu \mathrm{l}$ of single donor adult human serum on specially prepared glass coverslips (9). These round coverslips were held on a slide used for agglutination testing and placed inside a square Petri dish containing a gauze pad saturated with sterile water. The cells were allowed to adhere at $37^{\circ} \mathrm{C}$ for $60 \mathrm{~min}$. After the adherence step, $C$. albicans $(5 \mu \mathrm{l})$ was added at a BAM:Candida ratio of 2:1. After a 30-min ingestion period, the coverslips were aspirated and replenished with $30 \mu \mathrm{l}$ of BSH containing $20 \%$ adult human serum. The coverslips were incubated at $37^{\circ} \mathrm{C}$ for an additional $3.5 \mathrm{~h}$, fixed in $95 \%$ methanol for $10 \mathrm{~min}$, washed in deionized water, and stained with a $10 \%$ Giemsa solution. Our method is a variation of a slide assay that used the cytologic appearance of stained Candida within neonatal polymorphonuclear leukocytes to ascertain their killing and destruction (10). The coverslips were examined microscopically $(1000 \times)$ and 100 Candida ingested by BAM (those surrounded by cytoplasm and a halo of the phagolysosome) were scored according to the following criteria: 1) stained and filamented, 2) stained and unfilamented, 3) unstained and filamented, and 4) unstained and unfilamented (Fig. 1). Microscopic assessment of macrophages was limited to those phagocytes containing 1-4 yeast because preliminary studies indicated that BAM were less effective in killing and digesting yeast during higher phagocytic load. During the scoring of antiCandida activity, ingestion rates (number of BAM with intracellular Candida/100 BAM) and phagocytic profiles [number of intracellular yeast $(0,1,2,3,4,>4)$ /phagocytic macrophage] were determined. During scoring, extracellular yeast that remained on the coverslip after the 4 -h incubation period were scored as filamented or nonfilamented.

Adult versus normal newborn animal studies. These studies were undertaken to ascertain: 1) if BAM of animals produced findings similar to human neonates and adults and 2) if Mucomyst altered the microbicidal activity of these BAM. Twentyfour-hour-old (newborn, $n=7$ ) and 4-month-old New Zealand albino rabbits (adults, $n=4$, two of each sex), free of Bordetella bronchiseptica in the lower respiratory tract, were killed using either intracisternal injection of pentobarbital $(25 \mathrm{mg} / \mathrm{kg})$ or intravenous pentobarbital anesthesia $(50 \mathrm{mg} / \mathrm{kg})$ followed by an air embolus, respectively. The trachea was aseptically exposed, a sterile cannula was introduced and lung lavage was carried out as previously described (11). Lavage effluents were centrifuged

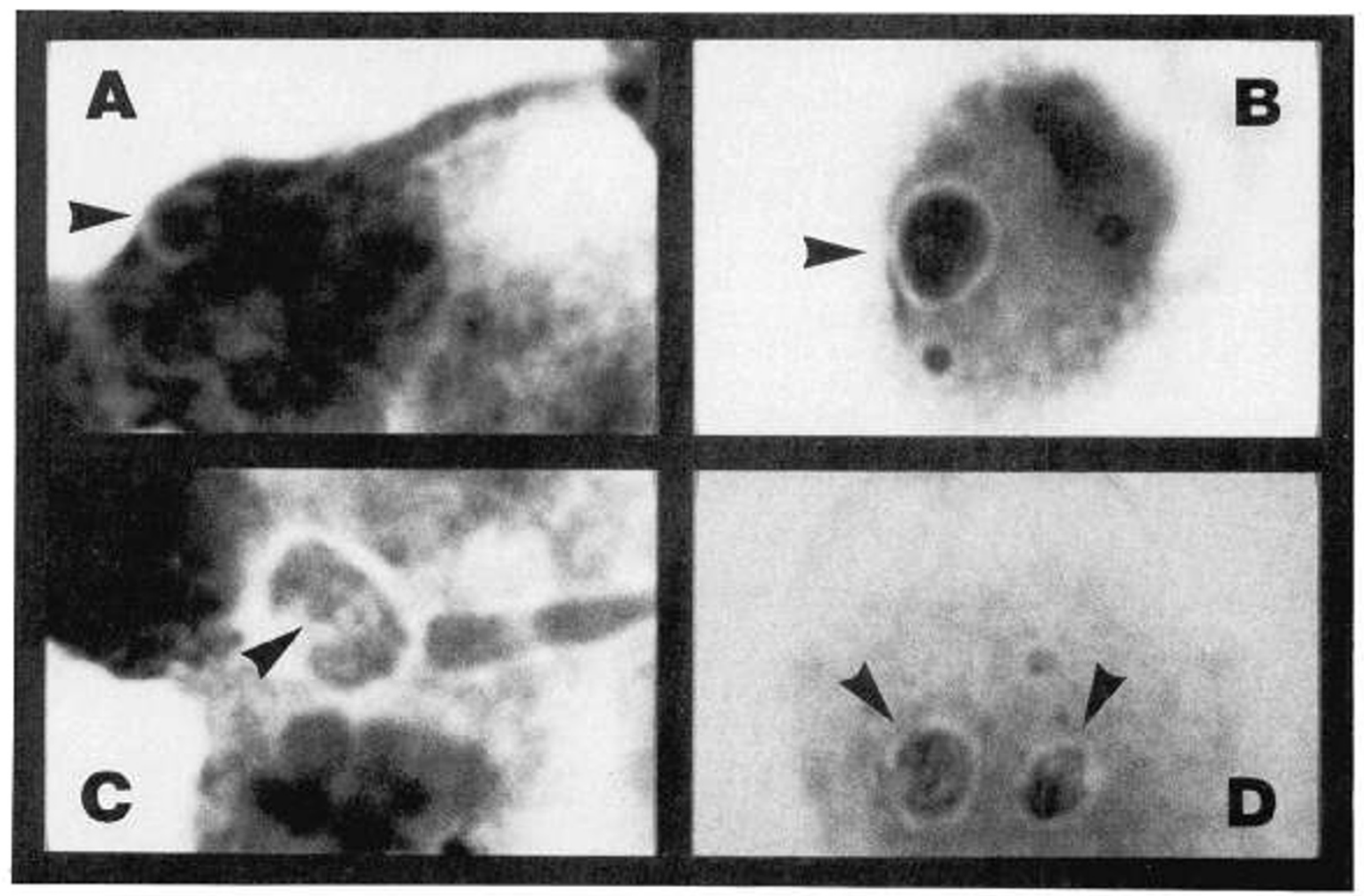

Fig. 1. Representative photomicrographs of $C$. albicans $4 \mathrm{~h}$ after mixing BAM and yeast in the in vitro anti-Candida slide assay. Ingested yeast were classified into one of the following four categories based on their growth and staining characteristics: $A$, stained and filamented yeast. The arrowhead indicates a parent yeast cell with a filamentous pseudogerm tube. This Candida stains deeply and uniformly by the Giemsa method. The germ tube means it has grown intracellularly after ingestion. The staining characteristics of the germ tube are not considered in the scoring. This parent yeast cell may be living or dead. $B$, stained and unfilamented yeast. The arrowhead indicates a yeast that is stained but has no germ tube. It has not grown since ingestion and may be in a living or dead cell. $C$, unstained and filamented yeast. Yeast that have been killed and are being destroyed have the following progressive staining appearances: 1) light and nonuniform staining (moth-eaten look), 2) a lucent band through the pole or equator of the parent yeast cell, or 3) the yeast cell is totally unstained (termed "ghosts"). The arrowhead indicates a yeast cell that has germinated intracellularly, has been killed and is being digested. D, unstained and unfilamented yeast. The arrowheads show two "moth-eaten" ingested yeast. These two fungi have not germinated after uptake. These Candida have been killed and are undergoing destruction. 
and the recovered cell pellets were divided into two portions that were resuspended either in RPMI + Mucomyst (as above) or RPMI alone. These suspensions were kept on ice for $2 \mathrm{~h}$. Thereafter, cells were pelleted by centrifugation $(150 \times g)$, resuspended in $\mathrm{BSH}$, and quantitated. After the concentration was adjusted to $5 \times 10^{6} \mathrm{BAM} / \mathrm{ml}$, the cells were used in the in vitro antiCandida assay that was outlined in the previous section.

Animal hyperoxia experiment. This experiment was undertaken to determine if oxygen exposure could induce further defects in newborn rabbit macrophage anti-Candida activity, thereby duplicating the deficiencies seen in the human neonates. Neonatal rabbit alveolar macrophages were isolated by lavage (without Mucomyst) from animals reared for $96 \mathrm{~h}$ in $95+\%$ oxygen $(n=8)$ or room air $(n=8)$. These conditions of exposure have been previously described (12). The cells harvested from two animals within each group were mixed together in order to provide sufficient macrophage numbers for slide assays at time points $0,1,2$, and $4 \mathrm{~h}$. During data analysis, $n=4$ was used as the number within each group (oxygen or room air), at each time point. Slide assays were scored as previously discussed.

Yeast kinetic analysis. The slide assays generated in the hyperoxia experiment were evaluated for the percent of extracellular yeast that were filamented ( 100 yeast counted/slide/time point) and for the percent of macrophages that were phagocytic (100 consecutive macrophages counted/slide/time point).

Data analysis. Anti-Candida activity of neonatal and adult groups was compared using the Mann-Whitney $U$ test with correction for Bonferroni inequality. When more than one assay was performed on human newborns during their period of intubation, the values were averaged and treated as a single mean score for rank sum analyses. The two-tailed paired $t$ test was used for animal studies that determined the effect of Mucomyst on the anti-Candida activity of the BAM. For data to be subjected to paired data analysis a probability plot of the data was first done to determine that the data was normally distributed. A statistical software package was used for these calculations (Systat, Systat Inc., Evanston, IL). Results are expressed as mean \pm SD. The significance level selected was $p<0.05$.

\section{RESULTS}

Patients. Gestational age of the human newborns from whom BAM were obtained ranged from $26-42 \mathrm{wk}$. Because our patients tended to be either very small premature infants or term neonates with congenital heart disease or pulmonary hypertension, the population was divided into a group under $1.2 \mathrm{~kg}[782 \pm 64 \mathrm{~g}$ $(8 / 22)$, mean $\pm \mathrm{SD}$ (no. patients/no. samples)] and a group above $1.2 \mathrm{~kg}[2693 \pm 860 \mathrm{~g} \mathrm{(16/32)}$ ] for the purpose of data analysis. Three of the 16 subjects in the $>1.2 \mathrm{~kg}$ group were in the $1.2-2.0 \mathrm{~kg}$ range. Because their anti-Candida activity was similar to that reported in studies with larger newborns, they were included in the heavier weight category. Thirteen of the 24 neonates were studied at more than one time point. The list of clinical diagnoses for the two weight categories is shown in Table 1.

Human data. No differences were found in the ingestion rate of C. albicans by human newborn and adult BAM $(<1.2 \mathrm{~kg}=$ $29 \pm 11 \%,>1.2 \mathrm{~kg}=29 \pm 10 \%$, and adults $=40 \pm 17 \%$, mean $\pm \mathrm{SD}$ ). Ingestion profiles indicated that phagocytic newborn and adult BAM also had similar mean numbers of intracellular Candida $(<1.2 \mathrm{~kg}=1.7 \pm 0.1,>1.2 \mathrm{~kg}=1.6 \pm 0.1$, and adults $=1.8 \pm 0.2$ ingested yeast $/$ phagocytic BAM, mean \pm SD). Less than $3 \%$ of newborn and adult BAM had ingested $>4$ yeast/ phagocytic BAM.

Human BAM recovered from healthy adults were five times more effective in restricting the intracellular filamentation or growth of $C$. albicans when compared to BAM aspirated from the airways of sick newborns (Fig. 2). This finding is in sharp contrast with the fact that $>97 \%$ of extracellular $C$. albicans had germinated after $4 \mathrm{~h}$ under conditions of the assay.
Table 1. Clinical conditions of neonatal subjects used to study anti-Candida activity of newborn $B A M$

\begin{tabular}{ccc}
\hline & $<1.2 \mathrm{~kg}$ & $>1.2 \mathrm{~kg}$ \\
Diagnosis & $(n=8)$ & $(n=16)$ \\
\hline
\end{tabular}

Prematurity ( $<37 \mathrm{wk})$

Respiratory distress syndrome

Patent ductus arteriosus

Bronchopulmonary dysplasia

Congenital heart disease

Primary pulmonary hypertension

of newborn

Severe apnea

Meconium aspiration

Renal failure

\begin{tabular}{ll}
8 & 6 \\
4 & 1 \\
5 & 2 \\
2 & 2 \\
0 & 8 \\
0 & 5 \\
& \\
2 & 1 \\
0 & 2 \\
1 & 2 \\
\hline
\end{tabular}

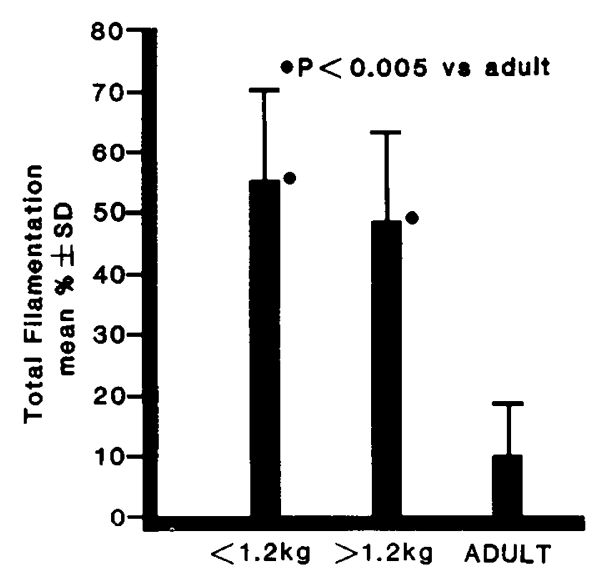

Fig. 2. Intracellular filamentation or growth of C. albicans after their uptake by human newborn or adult BAM. The histograms represent the mean number of $C$. albicans that had filamented (stained and unstained) per 100 yeast ingested by BAM.

However, Figure 3 shows that human newborn and adult BAM did not differ in their overall ability to kill and digest $C$. albicans. The apparent disparity between the observation that newborn BAM could not restrict intracellular growth of Candida compared to adult BAM while they achieved a total killing and destruction rate equal to the adult BAM may be explained as follows. Human neonatal BAM destroyed filamented Candida twice as well as ungerminated organisms (Fig. 3). Because approximately $50 \%$ of Candida ingested by neonatal lung macrophages germinated intracellularly, these phagocytes were able to destroy these fungi at a rate that was comparable to adult BAM. Adult BAM not only significantly restricted the intracellular growth of Candida (ca. $10 \%$ germinated intracellularly), but these mature phagocytes were twice as effective as neonatal BAM in killing and digesting ungerminated organisms (Fig. 3). Thus overall, adult and newborn BAM had total percentages of killed and digested Candida that were equivalent.

Normal newborn and adult animal data. Studies using alveolar macrophages recovered from normal 1-day-old and adult rabbits concur with our human studies (Fig. 4). Newborn rabbit BAM demonstrated substantially lower total killing and digestion, and increased yeast filamentation. In contrast to the human studies, both newborn and adult rabbit BAM demonstrated a reduced ability to kill and digest nonfilamented $C$. albicans versus filamented yeast $(p<0.001)$, with neonatal rabbit lung macrophages showing the greater impairment. N-acetyl-L-cysteine did not affect the ability of either newborn or adult rabbit BAM to kill and digest $C$. albicans (newborn BAM $=40 \pm 6 \%$ destroyed intracellular yeast versus newborn BAM + Mucomyst $=42 \pm$ $5 \%$ and adult $\mathrm{BAM}=56 \pm 5 \%$ killed and digested yeast versus adult BAM + Mucomyst $=67 \pm 10 \%$, mean \pm SD).

Hyperoxia experiment. There were no significant differences 


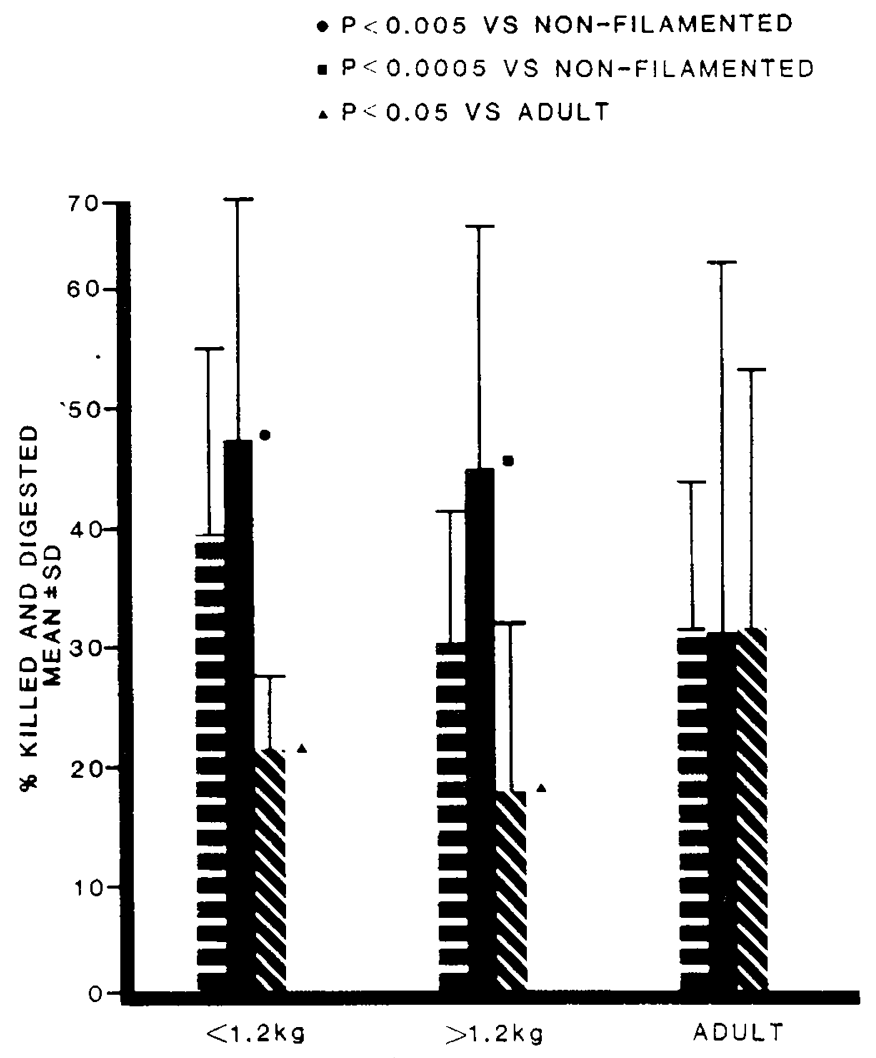

Fig. 3. Killing and digestion of C. albicans after phagocytosis by human newborn and adult BAM. The horizontally striped histogram shows the total percentage of ingested yeast that were killed and digested per 100 intracellular Candida. The solid histogram indicates the percentage of ingested and filamented Candida and the diagonally striped histogram displays the percentage of intracellular nonfilamented yeast that were destroyed per the total number of $C$. albicans that were either filamented or unfilamented within BAM, respectively. Data are presented as mean $\pm \mathrm{SD}$.

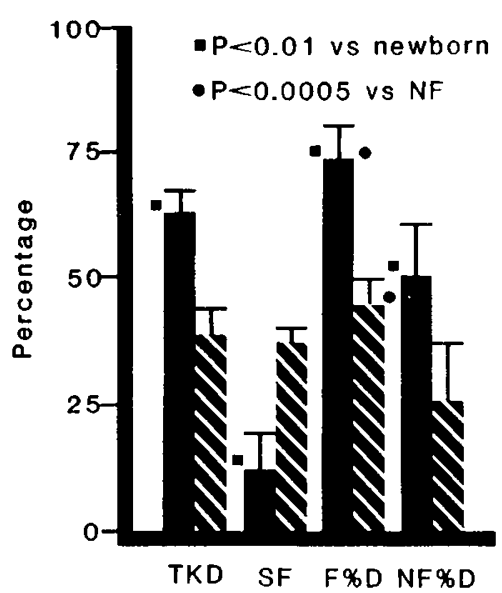

Fig. 4. Killing and digestion of $C$. albicans after uptake by rabbit neonatal and adult BAM. The solid histogram represents the adult macrophages and the diagonally striped histogram show the newborn cells. $T K D$, total killed and digested intracellular yeast/100 ingested Candida; $S F$, percentage of stained and filamented yeast $/ 100$ ingested Candida; $F \% D$, percentage of all intracellular filamented yeast that were killed and digested; $N F \% D$, percentage of all ingested, nonfilamented Candida that were inactivated and were being destroyed. Data are presented as mean $\pm \mathrm{SD}$.

in the percent of macrophages that were phagocytic between the oxygen and room air groups at any time points (Mann-Whitney $\mathrm{U}$ test), or between the different time points within each group (Friedman statistic). At the 4-h time point the oxygen group

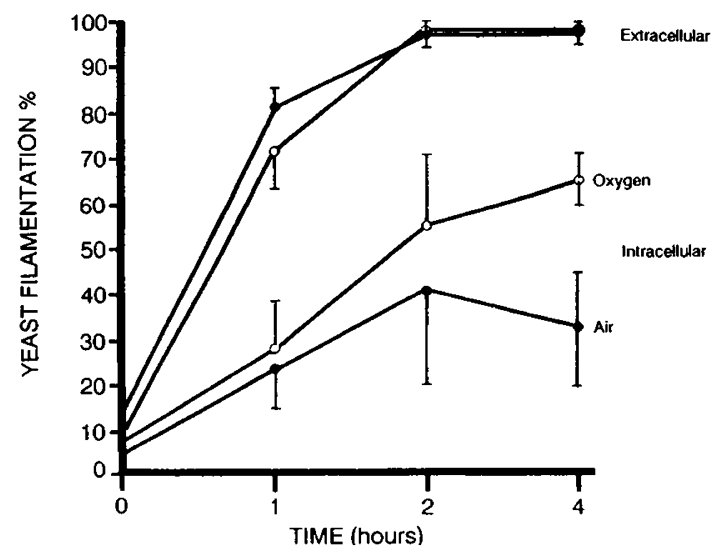

Fig. 5. Extracellular and intracellular yeast filamentation with time (h) in oxygen exposed (open circles) and room air exposed (closed circles) 4-day-old rabbits. Results shown as percentage of filamented yeast $/ 100$ yeast counted (mean $\pm \mathrm{SD}$ ) $p<0.025$ oxygen versus room air for intracellular yeast at $4 \mathrm{~h}$ (Mann-Whitney $\mathrm{U}$ test). $p<0.05$ for intracellular versus extracellular yeast (both in oxygen and room air groups) at $4 \mathrm{~h}$.

showed $43.75 \pm 10.81 \%$ of macrophages to be phagocytic whereas the room air group demonstrated $47.50 \pm 12.20 \%$ (mean $\pm \mathrm{SD})$.

Total killing and digestion of yeast (dead and filamented + dead and nonfilamented) increased with time in both the room air and oxygen groups. For the oxygen group total killing and digestion was $1.75 \pm 0.96 \%, 0.75 \pm 0.96 \%, 16.50 \pm 10.47 \%$, and $36.50 \pm 7.59 \%$ (mean \pm SD) at $0,1,2$, and $4 \mathrm{~h}$, respectively. The 2-h time point was significantly different from the 4-h time point ( $p<0.01$ by paired $t$ test). For the room air group the values were $2.0 \pm 2.16 \%, 2.75 \pm 3.09 \%, 17.0 \pm 7.83 \%$, and $38.75 \pm 6.70 \%$ (mean $\pm \mathrm{SD}$ ) at $0,1,2$, and $4 \mathrm{~h}$, respectively. The 2-h time point was significantly different from the 4-h time point ( $p<0.05$ by paired $t$ test). Oxygen exposure of newborn rabbit pups promoted intracellular yeast filamentation $(66.3 \pm$ $5.9 \%)$ compared to newborn animals raised in room air $(33.0 \pm$ $13.5 \%$ ) (mean $\pm \mathrm{SD}, p<0.025$, see Fig. 5). No differences were found in total killing and digestion between oxygen and room air groups.

Kinetic studies. Data for extracellular and total intracellular yeast filamentation are shown in Figure 5. There was a profound difference in the percentage of filamented yeast between the intracellular and the extracellular compartments. Although extracellular yeast were unrestricted in their ability to grow (filamentation $=98 \pm 0.5 \%$ by $2 \mathrm{~h}$ ), yeast ingested by macrophages from normal 4-day-old rabbits were limited to $33 \pm 13.5 \%$ filamentation (mean $\pm \mathrm{SD}, p<0.05$ by Mann-Whitney $\mathrm{U}$ test).

\section{DISCUSSION}

The method described here for the analysis of antifungal capacity of neonatal pulmonary macrophages has several unique advantages. Only a small number of cells is needed (minimum $1 \times 10^{5} /$ coverslip). The cells can be obtained during routine tracheal suctioning; therefore, no special invasive procedures are required. The suctioned material from intubated newborns contains predominantly macrophages, but small numbers of neutrophils and epithelial cells are present. By allowing adherence to glass coversiips, we selected for the bronchoalveolar macrophage. Contaminating undesired cell types and uningested yeast were aspirated off the coverslip after the adherence and ingestion period. Inasmuch as only ingested Candida were counted in the assay, the presence of other mononuclear cells capable of glass adherence, but not phagocytosis, did not affect the results. The presence of lymphocytes was noted only occasionally, and the Candida ingested by the rarely seen polymorphonuclear leukocyte were easily recognized, but not included in the scoring. 
Although our samples may have contained macrophages mainly from the larger airways, we believed it was unethical to lavage the lungs of ill neonates to obtain a higher yield of deep alveolar cells. Certainly, some of the cells under study were exiting the lung from more distal air spaces.

In general, during the first 3 days of life, the number of cells obtained was insufficient for analysis although the required amount could be recovered in a few infants. This finding is consistent with the known increase in the number of pulmonary macrophages that occurs in the first few weeks of life $(13,14)$. Initially, Mucomyst was not used during the collection of the macrophages; however, it soon became obvious that mucus was entrapping the lung macrophages, resulting in low cell yield. In the animal studies we found that Mucomyst had no detrimental effect on the slide assay results. However, its inclusion in the RPMI during specimen collection greatly enhanced cell yield. The use of normal adult serum allowed the neonatal cells to function optimally by supplying adequate amounts of opsonic factors known to be essential for phagocytosis (15). Previously, neonatal neutrophils have been shown to exhibit a defect in phagocytosis when neonatal serum was used as a source of opsonins; this defect was correctable by the use of adult serum (16). The use of a standard adult serum allowed us to focus on the microbicidal activity of the BAM themselves.

The finding that neonatal pulmonary macrophages were, on the whole, able to kill and destroy ingested $C$. albicans at a rate equal to adult BAM was unexpected. However, this finding was due to the fact that Candida entered a phase of active growth inside the neonatal BAM which rendered the yeast more susceptible to destruction. Adult human BAM effectively restricted filamentation, and killed and digested yeast regardless of their growth state within the phagocyte. However, unless intracellular growth had occurred, neonatal cells were not as effective in killing and digesting yeast. Our assay method is a modification of the tumbling and slide assays of Lehrer and associates $(8,10)$. In the assay described here, $C$. albicans were in log phase $(16 \mathrm{~h}$ of incubation) rather than a stationary phase of growth ( $48 \mathrm{~h}$ to 14 days of incubation) as reported with prior analytical methods. When older yeast are used for the assay, there is a prolonged period before they filament within phagocytes (17). C. albicans that are metabolically active and growing are killed more readily by nonoxidative effectors, namely cationic microbicidal peptides of rabbit and human phagocytes $(18,19)$. In vitro exposure of log phase and stationary phase Candida to hydrogen peroxide also reveal the younger yeast are more easily inactivated (Sherman $\mathrm{M}$, Lehrer $\mathrm{R}$, unpublished observations).

Using BAM recovered from 1-, 7-, and 28-day-old rabbits and a slide assay similar to our own, Zeligs et al. (20) reported that neonates may be prone to $C$. albicans infections because 1-dayold lung macrophages have a candidacidal defect. Human monocytes and BAM kill this opportunistic organism via $\mathrm{O}_{2}$-dependent and $\mathrm{O}_{2}$-independent mechanisms $(21,22)$. The relative contribution of each mechanism to Candida killing by human newborn BAM is unknown. Studies using neonatal BAM of rabbits have indicated that these cells have a reduced respiratory burst $\left(\mathrm{O}_{2^{-}}\right.$ dependent killing) and lower concentrations of lysosomal components $\left(\mathrm{O}_{2}\right.$-independent killing) than their adult counterparts (11, 23-25). The normal 1-day-old and adult rabbit BAM data support the concept that newborn rabbit lung macrophages have a developmental abnormality in microbicidal function. However, one cannot conclude that diminished microbicidal mechanisms with the human neonatal BAM are totally explained by maturational deficiencies.

The results of hyperoxia experiments in newborn rabbits are in agreement with the results obtained from human newborns. The animals exposed to oxygen demonstrated a defect in the ability to restrict intracellular yeast filamentation when compared to the animals raised in room air (Fig. 5). However, the overall ability to kill and digest was similar for the oxygen and room air groups. Many therapeutic interventions undertaken in the intensive care nursery, such as oxygen administration (26) and drug treatment $(27,28)$, may have caused the fungistatic defect noted in the human neonatal BAM. The data generated in the hyperoxia experiment, however, support the conclusion that oxygen exposure may be a principal agent in reducing fungistatic activity seen in the intubated human neonates. The newborn rabbit pups differed only in their oxygen exposure (room air versus $95 \%+0_{2}$ ) thereby removing the factors of positive pressure ventilation, intubation, drugs, and chronic lung disease. Pulmonary macrophages from oxygen-exposed animals demonstrated markedly diminished capacity to restrict intracellular yeast filamentation, and this finding is consistent with the adverse effects of oxygen exposure on the ability of the newborn rabbit BAM to kill ingested staphylococci (12).

Although total killing and digestion of yeast were similar in human neonates and adults, with similar findings in our animal experiments, the importance of intracellular yeast growth should not be ignored. Growing (filamenting) Candida were frequently seen to puncture the macrophage cell membrane. Such an occurrence would be expected to result in cell damage and death. Inasmuch as the process of microbial surveillance and defense is a dynamic one, the loss of a portion of the resident alveolar macrophage pool would be expected to result in diminished in vivo yeast clearance. Yeast whose intracellular germination had resulted in macrophage death would be released to the extracellular environment where reproduction would then continue.

It is entirely possible that BAM of healthy human newborns might function like normal adult lung macrophages, and BAM of ill, intubated adults might demonstrate the same abnormalities observed in the human neonatal cells in this study. The assay system described here may be useful in future studies as a means of delineating factors that make intubated patients highly susceptible to nosocomial pneumonia.

We conclude that the BAM recovered from intubated newborn infants undergoing intensive care have reduced capacity to restrict the intracellular growth of $C$. albicans. Oxygen therapy may be an important factor underlying this reduced fungistatic capacity.

Acknowledgments. The authors thank the nursing staff of our intensive care nursery for obtaining the neonatal specimens. We also appreciate the excellent technical assistance of Ms. Laurie Campbell.

\section{REFERENCES}

1. Jarvis WR 1987 Epidemiology of nosocomial infections in pediatric patients. Pediatr Infect Dis J 6:344-351

2. Hemming VG, Overall JC, Britt MR 1976 Nosocomial infections in a newborn intensive-care unit. N Engl J Med 294:1310-1316

3. LaGamma EF, Drusin LM, Mackles AW, Machalek S, Auld BAM 1983 Neonatal infections. An important determinant of late NICU mortality in infants less than $10000 \mathrm{~g}$ at birth. Am J Dis Child 137:838-841

4. Goldstein E, Lippert W, Warshauser D 1974 Bronchoalveolar macrophage. Defender against bacterial infection of the lung. J Clin Invest 54:519-528

5. Sherman M, Goldstein E, Lippert W, Wennberg R 1977 Neonatal lung defense mechanisms: a study of the alveolar macrophage system in neonatal rabbits. Am Rev Respir Dis 116:433-440

6. Suzuki Y, Lehrer RI 1980 NAD(P)H oxidase activity in human neutrophils stimulated by phorbol myristate acetate. J Clin Invest 66:1409-1418

7. Barbers RG, Gong Jr, Tashkin DP, Oishi J, Wallace JM 1987 Differential examination of bronchoalveolar lavage cells in tobacco, cigarette and marijuana smokers. Am Rev Respir Dis 135:1271-1275

8. Lehrer RI, Cline MJ 1969 Interaction of Candida albicans with human leukocytes and serum. J Bacteriol 98:996-1004

9. Shaw DR, Griffin FM 1981 Antibody-dependent and antibody-independent phagocytosis. In: Adams DO, Edelson PJ, Koren HS (eds) Methods For Studying Mononuclear Phagocytes. Academic Press Inc., New York, p 513

10. Oseas R, Lehrer RI 1978 A micromethod for measuring neutrophil candidacidal activity in neonates. Pediatr Res 12:828-829

11. Sherman MP, Lehrer RI 1984 Superoxide generation by neonatal and adult rabbit alveolar macrophages. J Leukocyte Biol 36:39-50

12. Sherman MP, Condiotti R 1987 Hyperoxia damages phagocytic defenses of neonatal rabbit lung. J Appl Physiol 62:684-690

13. Merritt TA, Stuard ID, Puccia J, Wood B, Edwards DK, Finkelstein J, Shapiro DL 1981 Neonatal tracheal cytology: classification during respiratory distress syndrome and bronchopulmonary dysplasia. J Pediatr 98:949-956

14. Alenghat E, Esterly JR 1984 Alveolar macrophages in perinatal infants. Pedi- 
atrics 72:221-223

15. Stossel TP 1973 Evaluation of opsonic and leukocyte function with a spectrophotometric test in patients with infection and with phagocytic disorders. Blood 42:121-130

16. Dossett JH, Williams RC Jr, Quie PG 1969 Studies on interaction of bacteria serum factors, and polymorphonuclear leukocytes in mothers and newborns. Pediatrics 44:49-57

17. Lehrer RI. 1981. Ingestion and destruction of Candida albicans. In: Adams DO, Edelson PJ, Koren HS (eds) Methods for Studying Mononuclear Phagocytes. Academic Press Inc., New York, pp 693-708

18. Selsted ME, Szklarek D, Ganz T, Lehrer RI 1985 Activity of rabbit leukocyte peptides against Candida albicans. Infect Immun 49:202-206

19. Ganz T, Selsted ME, Szklarek D, Harwig SSL, Daher K, Bainton DF, Lehrer RI 1985 Defensins. Natural peptide antibiotics of human neutrophils. J Clin Invest 76:1427-1435

20. Zeligs BJ, Nerurkar LS, Bellanti JA 1984 Chemotactic and candidacidal responses of rabbit alveolar macrophages during postnatal development and the modulating roles of surfactant in these responses. Infect Immun 44:379385

21. Lehrer RI 1975 The fungicidal mechanisms of human monocytes. I. Evidence for myeloperoxidase-linked and myeloperoxidase-independent candidacidal mechanisms. J Clin Invest 55:338-346
22. Catterall JR, Black CM, Leventhal JP, Rizk NW, Wachtel JS, Remington JS 1987 Nonoxidative microbicidal activity in normal human alveolar and peritoneal macrophages. Infect Immun 55:1635-1640

23. Nerurkar LS, Zeligs BJ, Bellanti JA 1977 Maturation of the rabbit alveolar macrophage during animal development. II. Biochemical and enzymatic studies. Pediatr Res 11:1202-1207

24. Sherman MP, Lehrer RI 1985 Oxidative metabolism of neonatal and adult rabbit lung macrophages stimulated by opsonized group B streptococci. Infect Immun 47:26-30

25. Ganz T, Sherman MP, Selsted ME, Lehrer RI 1985 Newborn rabbit alveolar macrophages are deficient in two microbicidal cationic peptides, MCP-1 and MCP-2. Am Rev Respir Dis 132:901-904

26. Zimmerman JJ 1986 Pharmacologic modulation by prostaglandin $E_{1}$ of superoxide anion production by human polymorphonuclear leukocytes. Crit Care Med 14:761-767

27. O'Neill SJ, Sitar DS, Klass DJ, Taraska VA, Kepron W, Mitenko PA 1986 The pulmonary disposition of theophylline and its influence of human alveolar macrophage bactericidal function. Am Rev Respir Dis 134:12251228

28. Mammel MC, Fitterman C, Coleman M, Boros SJ 1987 Short-term dexamethasone therapy for bronchopulmonary dysplasia: acute effects and 1-year follow-up. Dev Pharmacol Ther 10:1-11

\section{Richard D. Rowe Award}

The Richard D. Rowe Award in Perinatal Cardiology has been established by the colleagues, trainees, and friends of Dr. Rowe to mark his many achievements in this field. Dr. Rowe was very proud of the award. While all of us are deeply saddened by his sudden illness and death, we hope the qualities everyone admired-his deep and unflinching commitment to excellence in practice, research, and training; his strength in crisis, his tremendous integrity, and, above all, his humility-will serve as models for the coming generation of physicians and scientists.

The award is a prize of $\$ 750$ to be presented annually at the meeting of the Society for Pediatric Research for the best work by a young investigator within three years of completing postdoctoral MD or $\mathrm{PhD}$ fellowship training. The award was judged this year on the basis of the abstract and presentation; but in the future, submission of a short paper, a curriculum vitae, and a letter from the sponsor also will be required.

Dr. Einat Birk is the 1988 winner of the Richard D. Rowe Award. She is a fellow of Dr. Abraham Rudolph at the Cardiovascular Research Institute, University of California, San Francisco. Her presentation was titled: Fetal thyroidectomy reduces postnatal myocardial beta adrenergic receptor responses in newborn lambs. Coauthors were Drs. Abraham Rudolph and James Roberts. 